\title{
Predição de dados agronômicos em goiabeiras e separação de alvos por meio de veículo aéreo não tripulado
}

Prediction of agronomic data in guava trees and target separation by unmanned aerial vehicle

\author{
P. R. Giongo ${ }^{1 *}$; J. D. Orlandini ${ }^{1}$; B. H. T. Arantes ${ }^{2}$; L. F. Gomes 3 ; V. H. Moraes ${ }^{3}$; \\ A. R. Costa $^{1}$; A. A. Ribon ${ }^{4}$ \\ ${ }^{1}$ Universidade Estadual de Goiás, 75920-000, Santa Helena de Goiás-GO, Brasil \\ ${ }^{2}$ Universidade Federal do Rio Grande do Sul, 91501-970, Porto Alegre - RS, Brasil \\ ${ }^{3}$ Instituto Federal Goiano, 75906-785, Rio Verde - GO, Brasil \\ ${ }^{4}$ Universidade Estadual de Goiás, 76190-000, Palmeiras de Goiás-GO, Brasil \\ *pedro.giongo@ueg.br \\ (Recebido em 10 de outubro de 2019; aceito em 02 de abril de 2020)
}

\begin{abstract}
A agricultura digital, a aquisição e análise de informações remotas dos dados vem se tornando frequente com o uso dos Veículos Aéreos Não Tripulados (VANTs) e miniaturização dos sistemas de sensores. Com isto, esta pesquisa teve como objetivo, analisar o uso do sensoriamento remoto para a predição de variáveis agronômicas como diâmetro de caule, altura de planta e área de copa de goiabeiras, bem como para a diferenciação de alvos predominantes na área de estudo. Em função dos resultados, é possível com o sensoriamento remoto, determinar-se a área de copa, altura de planta e diâmetro de caule. Além disso o sensoriamento remoto permite a separação das plantas de goiabas, plantas daninhas, solo exposto, sombra de plantas e de palhada, selecionando de forma adequada as faixas espectrais ideias para a diferenciação de cada alvo.

Palavras-chave: Agricultura de precisão, sensoriamento remoto, pomar.
\end{abstract}

Digital agriculture, remote data acquisition and analysis has become commonplace through the use of unmanned aerial vehicles (UAVs) and miniaturization of sensor systems. Thus, this research aimed to analyze the use of remote sensing for the prediction of agronomic variables such as stem diameter, plant height and guava canopy area, as well as for the differentiation of predominant targets in the study area. Depending on the results, it is possible with remote sensing to determine canopy area, plant height and stem diameter. In addition, remote sensing allows the separation of guavas plants, exposed soil, plant shade and mulch orchards, appropriately selecting the ideal spectral bands for differentiation of each target.

Keywords: Precision farming, remote sensing, orchard.

\section{INTRODUÇÃO}

A agricultura digital para a aquisição de informações de forma remota e análise dos dados tem se tornando frequente com o uso dos Veículos Aéreos Não Tripulados (VANTs) e a miniaturização dos sistemas de sensores. Ao contrário dos sistemas orbitais, para menores áreas os sistemas aéreos não tripulados permitem a aquisição de imagens com maior resolução espacial e temporal e com menor custo, de suma importância a agricultura $[1,2]$. A potencialidade da agricultura digital está no benefício de melhor tomada de decisões nas indústrias agrícolas, trazendo maiores ganhos em produtividades e sustentabilidade [3 - 5] com o uso de orientação do sistema de posicionamento global, mapeamento de produção e aplicações a taxa variada $[6,7]$.

$\mathrm{O}$ sensoriamento remoto aplicado em pomares permite a identificação da variabilidade na produção através das respostas espectrais [8], o que direciona e auxilia as amostragens de solo em campo e a identificação de pomares com baixa fitossanidade. Além disso, com o conhecimento do rendimento agrícola, o agendamento da colheita, o número de colhedores, o armazenamento e a logística, podem ser organizadas com antecedência. A diferença do número de frutos por plantas pode estar relacionada a diversos fatores como: estrutura do solo, disponibilidade de água e nutrientes, competição com outras plantas, manejo utilizado, clima de uma região [9], além da incidência de pragas e doenças. 
Nesse contexto há diversos estudos sobre modelos de estimativa de produtividade de pomares em diversas culturas agrícolas utilizando parâmetros estruturais, fisiológicos e espectrais, tais como área transversal de tronco, área foliar, volume do dossel, imagens térmicas, nuvens de pontos e o uso de imagens em geral [10 - 21].

Em paralelo, na agricultura, para manter a qualidade e a quantidade da produção agrícola é necessária na maioria das vezes o uso de herbicidas. Entretanto a aplicação de herbicidas em locais que não apresenta a presença de plantas invasoras tende a aumentar os custos de aplicação e diminuir até mesmo o rendimento e a sustentabilidade [22, 23]. Com o sensoriamento remoto e a avaliação do comportamento espectral é possível a obtenção de mapa de cobertura de planta daninhas, permitindo uma aplicação direcionada $[24,25]$.

Superando as resoluções espectrais e temporais dos satélites, os VANTs permitem que as plantas daninhas de pequenas dimensões sejam identificadas e acompanhadas ao longo do tempo com maior precisão. Casos de sucesso no mapeamento de plantas daninhas com o uso da classificação supervisionada foi encontrado no trabalho de Pérez-Ortiz et al. (2015) [26], bem como na pesquisa de Castaldi et al. (2017) [27], para a diferenciação de milho e daninhas, cuja levaram uma diminuição do uso de herbicidas. Já existem pesquisas que avaliaram a escala adequada para o mapeamento de manchas de plantas invasores, bem como a configuração e a especificação de um drone para o manejo precoce $[28,29]$.

Há ainda de se considerar em imagens aéreas os efeitos negativos da sombra das nuvens relatado por alguns autores, se refere a mudança de reflectância na superfície, erro na classificação de uso do solo, dificuldade de se trabalhar com índices de vegetação e de detecção de mudanças [30, 31]. Além disso, Hsieh et al. (2016) [32] em sua pesquisa constatou que os valores dos números digitais dos pixels sombreados são diferentes daqueles não sombreados.

Neste contexto, esta pesquisa teve como objetivo analisar a relação do uso das bandas do infravermelho próximo (Nir), verde e azul e de alguns índices de vegetação do sensor Agrocan Geo, a área de copa, altura de planta e diâmetro de caule em pomares de goiabeiras, além de determinar qual o melhor modelo empírico para estimar tais variáveis agronômicas citadas. Ainda objetivou avaliar a separação dos alvos espectrais predominantes presente na área de estudo utilizando as bandas Nir do Agrocan Geo e verde, vermelha e azul do sensor RGB do phantom.

\section{MATERIAL E MÉTODOS}

\section{1 ÁREA E OBJETO DE ESTUDO}

Os dados de campo desta pesquisa foram obtidos em pomar de goiaba (17 40'15.82"S"; $50^{\circ} 32$ '21.30"O), localizado no município de Santa Helena de Goiás, no estado de Goiás. As plantas estavam com a idade de 4 a 6 anos. O espaçamento de plantio da área é de 6 metros entre linhas e 4 metros entre plantas (Figura 1).

Foram coletados em campo dados de diâmetro de caule e altura de planta e, na imagem de drone, a área de copa para um conjunto de 19 plantas selecionadas (Figura 1) denominados neste trabalho de dados agronômicos. Para a captura das imagens foi utilizado um drone phantom 4 advanced, com um sensor RGB e uma câmera Agrocan Geo com as bandas verde, azul e infravermelho próximo (Nir). As coletas em plantas selecionadas foram realizadas de forma aleatória. Um paquímetro foi utilizado para a coleta de diâmetro de caule, as imagens para o cálculo de área de copa e uma mira topográfica milimetrada para a medição de altura de planta. 


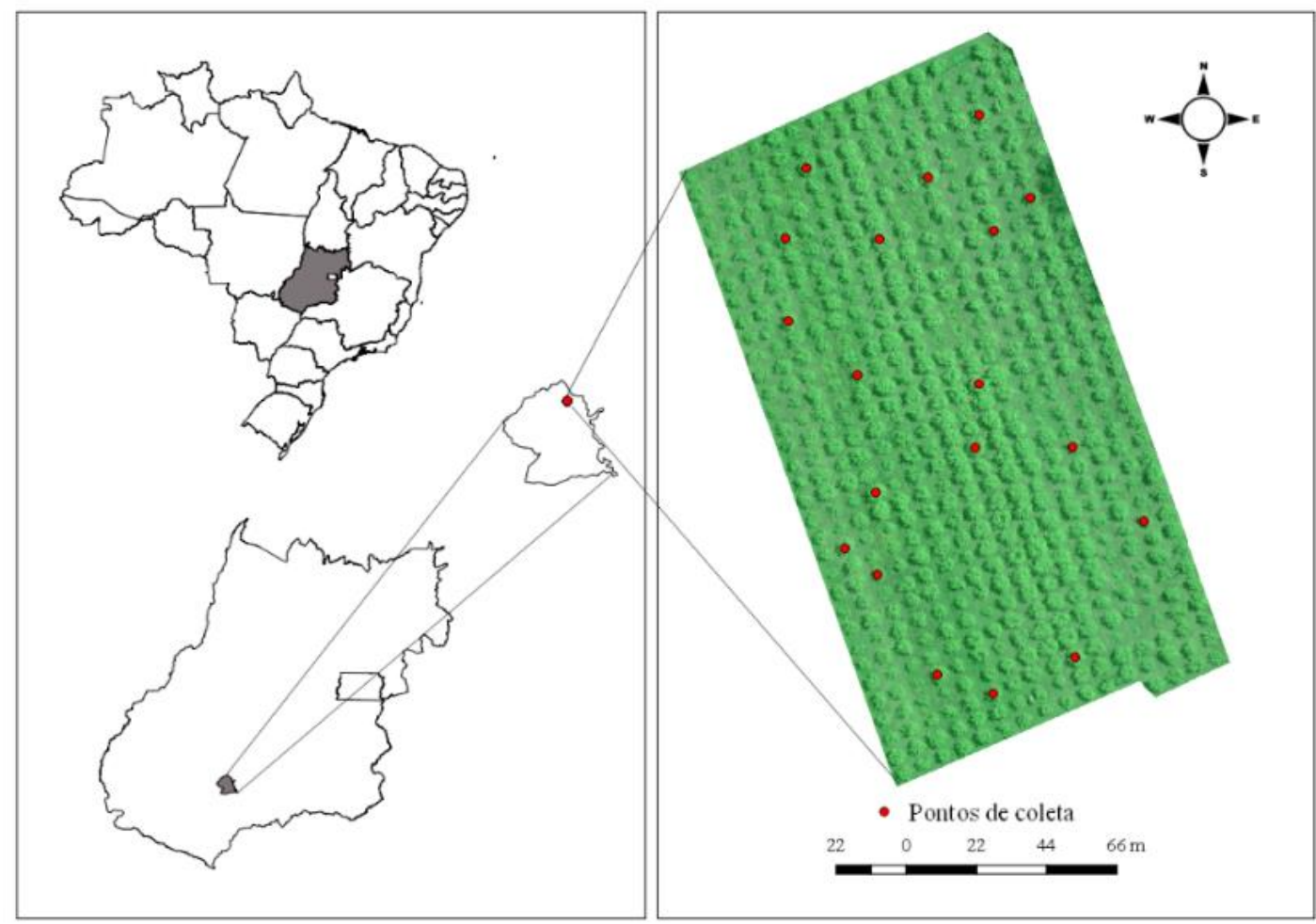

Figura 1: Mapa do Brasil com destaque para o estado de Goiás e o município de Santa Helena de Goiás. Em vermelho estão indicados os pontos de coleta de plantas selecionadas em pomar de goiabeira.

O plano de voo foi programado no aplicativo DroneDeploy, que é gratuito e compatível com o drone utilizado. As imagens foram processadas no software Pix $4 D$, no laboratório de Vants e Drones na Agricultura (LVDA) do Instituto Federal Goiano em Rio Verde. A extração de informações espectrais e a obtenção de índices de vegetação (Tabela 1), foram realizadas com uso do software $Q G I S$ v. 3.4 e as análises estatísticas no Minitab. Todos os índices foram gerados com a câmera Agrocan Geo e escolhidos em função de serem comuns e listados na literatura deste trabalho.

Tabela 1: Índices de vegetação (IV) aplicados ao pomar de goiabeiras com imagens de drone no município de Santa Helena de Goiás, Goiás.

\begin{tabular}{ccc}
\hline IV & Nome & Equação - bandas \\
\hline IV1 & BNDVI & (Nir - azul) / (Nir + azul) \\
\hline IV2 & GNDVI & (Nir - verde) / (Nir + verde) \\
\hline IV3 & Nir / Verde & Nir / verde \\
\hline IV4 & Nir / Azul & Nir / azul \\
\hline
\end{tabular}

\subsection{MÉTODOS}

\subsubsection{Coleta de dados agrômicos e realização do voo}

Para medição direta do diâmetro do caule, usou-se um paquímetro digital e foi medida a $20 \mathrm{~cm}$ de altura do solo. A altura de planta foi obtida do nível do solo até o ramo mais alto e essa medida foi executada com uma régua milimetrada utilizada em levantamentos topográficos, de modo que ela estivesse mais nivelada possível. A área de copa foi extraída através do ortomosaico das imagens do drone.

O voo foi executado no dia 08/09/2018 às 9:00 da manhã, em dia de céu claro, evitando assim a presença de nuvens e minimizando o efeito de sombra em função da inclinação do sol sobre os pomares. 
Por conseguinte, usou-se uma altura de voo de 100 metros, com uma sobreposição de imagens de $80 \%$ longitudinal e $80 \%$ lateral. Tal sobreposição utilizada tinha o objetivo prevenir falhas entre faixas consecutivas, como resultado da deriva, inclinações, variação de altura do voo e do comportamento do terreno, além de permitir uma boa construção de mosaico e geração de pontos de apoio por fototriangulação de imagens.

\subsubsection{Geração do ortomosaico e extração de informações do pixel para futuras regressões}

No software Pix4D as imagens foram processadas para a obtenção do ortomosaico. No processamento, o software busca informações armazenadas no arquivo de log de cada imagem, para que o ortomosaico seja gerado. Não foi utilizado painel de calibração durante o voo.

Utilizando o software Qgis, foi extraído uma média dos valores dos pixels de cada banda, representando o comportamento espectral de cada ponto para as plantas e alvos selecionados. Nesta metodologia utilizou-se somente o sensor Agrocan Geo. Por conseguinte, para o cálculo da média utilizou-se uma circunferência de 1,5 metros de raio. A ferramenta utilizada para a obtenção da média, foi a estatística por zona. A fim de evitar os efeitos da sombra, em função da inclinação do sol sobre o valor médio dos pixels de cada planta, uma classificação supervisionada foi aplicada para a eliminação destes pixels. Os índices de vegetação foram obtidos através da calculadora raster do Qgis.

As bandas Nir, verde e azul, foram renomeados nas tabelas dos resultados e discussões como Nir, G e B respectivamente. Para os índices de vegetação BNDVI, GNDVI, NIR/Verde e NIR/Azul respectivamente como IV1, IV2, IV3 e IV4 (Tabela 1). A área de copa como AC, o diâmetro de caule como DC e altura de planta como AP.

\subsubsection{Separação de alvos}

Para possibilitar a separação dos alvos presentes na área levantada, o comportamento espectral de cada um deles foi definido para as bandas vermelha, verde, azul e Nir. As bandas da faixa do visível utilizadas são do sensor RGB e a Nir do Agrocan Geo. Dos elementos predominantes encontrados em meio ao pomar tem-se 6 tipos: 3 tipos de plantas daninhas, a sombra das goiabeiras, a palhada e o solo exposto. Das plantas daninhas, duas delas estavam em boa situação de fitossanidade, enquanto a outra estava senescendo devido a aplicação de herbicida.

Para o cálculo do comportamento espectral de cada alvo propriamente dito, a resposta espectral de vários pixels fora coletada de cada banda relativo aos alvos, dentro do ortomosaico. Por conseguinte, uma média de todos os pixels amostrados foi realizada por banda e por alvo identificado.

\subsubsection{Análise estatística}

Para a escolha dos melhores modelos para estimar altura de planta, diâmetro de caule e área de copa em função das respostas espectrais e variáveis agronômicas, as análises estatísticas foram apoiadas na regressão dos melhores subconjuntos, cuja fornece informações sobre o ajuste de diferentes padrões permitindo selecionar um modelo com base em $\mathrm{R}^{2}, \mathrm{R}^{2}$ ajustado, $\mathrm{R}^{2}$ predito e $c p$ de Mallows.

Modelos com uma variável independente, $\mathrm{o} \mathrm{R}^{2}$ foi adotado para a explicar a variabilidade dos dados. Todavia, com modelos com mais de uma variável preditora, adotou-se o $\mathrm{R}^{2}$ ajustado, uma vez que ajusta o número de variáveis independentes com relação ao número de observações, apesar do $\mathrm{R}^{2}$ predito ser calculado com as observações que não estão incluídas no cálculo do modelo [33].

Como a regressão dos melhores subconjuntos seleciona as duas equações com os melhores $\mathrm{R}^{2}$ para cada número de preditora, foram selecionados modelos que apresentassem um $p$ valor menor que 0,10 a todas as variáveis independentes, aquelas que não apresentassem preditoras altamente correlacionadas entre si visualizada por meio do VIF (fatores de inflação de variância) e que os resíduos apresentassem um comportamento normal. Foi priorizado para escolha do melhor modelo, o menor $c p$ de Mallows. Além disso, foi adotado como limite para esta estatística um número máximo de 4 preditoras, tal que acima disso o modelo passa a apresentar um nível de significância 
pior que 0,90 , sendo assim a multicolinearidade deve ser evitada, uma vez que pode aumentar a variância dos coeficientes de regressão, de forma a tornarem inadequados [33].

$\mathrm{Na}$ tabela dos modelos dos melhores subconjuntos, é apresentado o maior valor de $c p$ de Mallows, de VIF, e de significância encontrados nas preditoras do modelo. O teste de normalidade foi realizado somente nos modelos que atendeu ao nível de significância estabelecido neste trabalho.

\section{RESULTADOS E DISCUSSÃO}

\subsection{Modelos empíricos para a determinação de altura de planta, diâmetro de caule e área de copa}

Com a estatística da regressão dos melhores subconjuntos e o uso do sensoriamento remoto, foi possível determinar as equações com melhores ajustes para determinação da altura das plantas do pomar em função de seu nível de significância e outros critérios. A um nível de significância a 5\%, a altura pode ser estimada pela área de copa com um $\mathrm{R}^{2}$ de $48,2 \%$ e pelo diâmetro de caule com $\mathrm{R}^{2}$ de $41,7 \%$, ressaltando que a área de copa foi extraída por uma ferramenta de cálculo de área diretamente das imagens. A medida de área de copa dos pomares obtidos via $Q g i s$ tende a apresentar uma precisão maior do que medidas de campo, tal que na imagem os ramos podem ser delimitados de forma correta devido à alta resolução espacial das imagens provenientes dos drones. $\mathrm{O}$ erro na medida de pequenas áreas com o uso de imagens sem pontos de apoio é de aproximadamente 5\% [34]. Quando se combina a resposta espectral das bandas com as variáveis agronômicas pode apresentar um $\mathrm{R}^{2}$ ajustado de $64,8 \%$, caso do uso de área de copa, diâmetro de caule, banda do azul e o índice de vegetação que utiliza a banda do Nir dividida pela banda do azul (IV4) (Tabela 2).

Apesar da equação 7 apresentada na Tabela 2 ser melhor do que a regressão linear simples, ela foi significativa a $10 \%$ e apresentou um VIF de 1,75. Se tal modelo for utilizado, é importante verificar a discrepância entre os dados preditos e pontos de verificação em campo, por meio do erro médio quadrático (RMSE). De acordo com Deroco et al. (2017) [35], seu modelo de predição por meio de uma regressão linear múltipla com um $p$-valor de uma das variáveis independentes de 0,08 apresentou um RMSE melhor do que uma regressão linear simples com um nível de significância a $5 \%$, o que indica que o $p$-valor acima de 0,05 e abaixo de 0,10 pode trazer bons resultados.

Todos os modelos com um nível de significância menor que 0,10 apresentou a distribuição normal dos resíduos. Por outro lado, o $c p$ de Mallows do melhor modelo de regressão linear múltipla foi baixo $(1,9)$, indicando uma pequena variância [36] (Tabela 2).

Tabela 2: Modelos empíricos para determinação de altura de planta de goiabeiras, por meio de dados agronômicos e espectrais, no município de Santa Helena de Goiás, Goiás.

\begin{tabular}{|c|c|c|c|c|c|c|c|c|c|c|c|c|c|c|c|c|}
\hline $\mathbf{E q}$ & $\mathbf{R}^{2}$ & $\begin{array}{l}\mathbf{R}^{2} \\
\text { (aj) }\end{array}$ & $\begin{array}{c}\mathbf{R}^{2} \\
(\mathbf{p r})\end{array}$ & $\begin{array}{c}\text { Cp de } \\
\text { Mallows }\end{array}$ & VIF & $p$ & $\begin{array}{c}\text { Teste de } \\
\text { Normalidade }\end{array}$ & DC & $\mathbf{A C}$ & Nir & $\mathbf{G}$ & B & IV1 & IV2 & IV3 & IV4 \\
\hline 2 & 44,9 & 41,7 & 30 & 6,9 & - & 0,0020 & Normal & $\mathrm{x}$ & & & & & & & & \\
\hline 4 & 52,8 & 46,9 & 23,2 & 5,8 & $>5$ & $>0,10$ & - & & $\mathrm{x}$ & & & & & & $\mathrm{x}$ & \\
\hline 5 & 66,2 & 59,5 & 45 & 2,4 & 1,64 & $>0,10$ & - & $\mathrm{x}$ & $\mathrm{x}$ & & & $\mathrm{x}$ & & & & \\
\hline 6 & 64 & 56,8 & 41,5 & 3,3 & 1,57 & $>0,10$ & - & $\mathrm{x}$ & $\mathrm{x}$ & & & & $\mathrm{x}$ & & & \\
\hline 8 & 72,3 & 64,4 & 54,9 & 2 & 2,20 & $>0,10$ & - & $\mathrm{x}$ & $\mathrm{x}$ & & $\mathrm{x}$ & $\mathrm{x}$ & & & & \\
\hline
\end{tabular}

Para a determinação do diâmetro de caule, nenhum modelo matemático de regressão múltipla com um nível de significância a $5 \%$ foi encontrado. Entretanto, pode-se predizer o diâmetro de caule com um $p$-valor de 0,057 combinando a altura de planta e a banda do azul. Os seus valores de $c p$ de Mallows e VIF foram adequados indicando a inexistência de relação entre a variáveis altura de planta e banda do azul, além de ter uma baixa variância dos dados. O R ${ }^{2}$ ajustado foi de $50,9 \%$, sendo este um modelo melhor a nível de coeficiente de determinação (equação 1) e é utilizada a AP para determinar DC com um $\mathrm{R}^{2}$ de 44,9 (Tabela 3). 
Tabela 3: Modelos empíricos para determinação do diâmetro de caule no pomar de goiaba no município de Santa Helena de Goiás, Goiás.

\begin{tabular}{|c|c|c|c|c|c|c|c|c|c|c|c|c|c|c|c|c|}
\hline $\mathbf{E q}$ & $\mathbf{R}^{2}$ & $\begin{array}{c}\mathbf{R}^{2} \\
(\mathbf{a j})\end{array}$ & $\begin{array}{c}\mathbf{R}^{2} \\
(\mathbf{p r})\end{array}$ & $\begin{array}{c}\text { Cp de } \\
\text { Mallows }\end{array}$ & VIF & $p$ & $\begin{array}{c}\text { Teste de } \\
\text { Normalidade }\end{array}$ & $\mathbf{A P}$ & $\mathrm{AC}$ & Nir & $\mathbf{G}$ & B & IV1 & IV2 & IV3 & IV4 \\
\hline 1 & 44,9 & 41,7 & 31,3 & 1,5 & - & 0,0020 & Normal & $\mathrm{x}$ & & & & & & & & \\
\hline 2 & 32,3 & 28,3 & 17,2 & 5,3 & - & 0,0110 & Normal & & $\mathrm{x}$ & & & & & & & \\
\hline 3 & 56,4 & 50,9 & 34,5 & 0,1 & 1 & 0,0570 & Normal & $\mathrm{x}$ & & & & $\mathrm{x}$ & & & & \\
\hline 4 & 52,2 & 46,2 & 28,9 & 1,4 & 1,02 & $>0,10$ & - & $\mathrm{x}$ & & & & & $\mathrm{x}$ & & & \\
\hline 5 & 59,6 & 51,5 & 20,1 & 1,1 & 1,79 & $>0,10$ & - & $\mathrm{x}$ & & & & $\mathrm{x}$ & & & $\mathrm{x}$ & \\
\hline 6 & 59,4 & 51,3 & 20,7 & 1,2 & 2,17 & $>0,10$ & - & $\mathrm{x}$ & & & $\mathrm{x}$ & $\mathrm{x}$ & & & & \\
\hline 7 & 63,3 & 52,9 & 7,5 & 2 & 2,88 & $>0,10$ & - & $\mathrm{x}$ & & $\mathrm{x}$ & $\mathrm{x}$ & & $\mathrm{x}$ & & & \\
\hline 8 & 62,4 & 51,6 & 3 & 2,3 & 2,37 & $>0,10$ & & $\mathrm{x}$ & & $\mathrm{x}$ & & & $\mathrm{x}$ & & $\mathrm{x}$ & \\
\hline
\end{tabular}

A área de copa é a única variável dependente que pode ser estimada de forma precisa com o uso do sensoriamento remoto. Entretanto, por meio de outra metodologia, a melhor forma de se determinar a área de copa de um pomar é através da altura de planta que apresenta um $\mathrm{R}^{2}$ de $51 \%$ e um $c p$ de mallows adequado para a equação (Tabela 4).

Tabela 4: Modelos empíricos para determinação de área de copa no pomar de goiaba no município de Santa Helena de Goiás, Goiás.

\begin{tabular}{|c|c|c|c|c|c|c|c|c|c|c|c|c|c|c|c|c|}
\hline $\mathbf{E q}$ & $\mathbf{R}^{2}$ & $\begin{array}{l}\mathbf{R}^{2} \\
\text { (aj) }\end{array}$ & $\begin{array}{c}\mathbf{R}^{2} \\
(\mathbf{p r})\end{array}$ & $\begin{array}{c}\text { Cp de } \\
\text { Mallows }\end{array}$ & VIF & $\underset{\text { valor }}{\mathbf{p}}$ & $\begin{array}{c}\text { Teste de } \\
\text { Normalidade }\end{array}$ & DC & $\mathbf{A P}$ & Nir & $\mathbf{G}$ & B & IV1 & IV2 & IV3 & IV4 \\
\hline 1 & 51 & 48,2 & 41,6 & $-1,2$ & - & 0,0010 & Normal & & $\mathrm{x}$ & & & & & & & \\
\hline 2 & 32,3 & 28,3 & 16,9 & 4 & - & 0,0110 & Normal & $\mathrm{x}$ & & & & & & & & \\
\hline 3 & 55,5 & 49,9 & 36,5 & $-0,5$ & 1,02 & $>0,10$ & - & & $\mathrm{x}$ & & & & & & & $\mathrm{x}$ \\
\hline 4 & 55,1 & 49,5 & 33,1 & $-0,4$ & 1,02 & $>0,10$ & - & & $\mathrm{x}$ & & & & $\mathrm{x}$ & & & \\
\hline 5 & 64,8 & 57,8 & 43,8 & $-1,1$ & 2,05 & $>0,10$ & - & & $\mathrm{x}$ & & & & $\mathrm{x}$ & $\mathrm{x}$ & & \\
\hline 6 & 63,2 & 55,8 & 37,6 & $-0,6$ & 2,17 & $>0,10$ & - & & $\mathrm{x}$ & & & & $\mathrm{x}$ & & $\mathrm{x}$ & \\
\hline 7 & 65,2 & 55,3 & 34,4 & 0,8 & 30,85 & $>0,10$ & - & & $\mathrm{x}$ & & & & $\mathrm{x}$ & $\mathrm{x}$ & $\mathrm{x}$ & \\
\hline 8 & 65,1 & 55,1 & 33,8 & 0.8 & 16,25 & $>0,10$ & - & & $\mathrm{x}$ & & & & $\mathrm{x}$ & $\mathrm{x}$ & & $\mathrm{x}$ \\
\hline
\end{tabular}

$\mathrm{Eq}=\mathrm{n}^{\mathrm{o}}$ da equação; $\mathrm{R}^{2}=$ coeficiente de determinação; $\mathrm{R}^{2}(\mathrm{aj})=$ coeficiente de determinação ajustado; $\mathrm{R}^{2}(\mathrm{pr})=$ coeficiente de determinação predito; $p=$ nível de significância; $\mathrm{DC}=$ diâmetro de copa; $\mathrm{AP}=$ altura de planta; $\mathrm{G}=$ banda do verde; $\mathrm{B}=$ banda do azul; IV1 = BNDVI; IV2 = GNDVI; IV3 = Nir / Verde; IV4 = Nir / Azul.

Para todos os modelos obtidas pela regressão dos melhores subconjuntos, tem-se as equações na Tabela 5. Em negrito tem-se os melhores $\mathrm{R}^{2}$ ajustado para a predição de cada variável agronômica.

Tabela 5: Modelos empíricos com as regressões dos melhores subconjuntos para altura de planta, diâmetro de caule e área de copa em pomar de goiabeira no município de Santa Helena de Goiás, Goiás.

\begin{tabular}{|c|c|}
\hline Modelo empírico & Equação matemática \\
\hline \multirow{4}{*}{ Altura de planta } & $\mathrm{y}=1,93+0,05482 \times \mathrm{AC}$ \\
\hline & $\mathrm{y}=1,21+0,2151 \times \mathrm{DC}$ \\
\hline & $\mathrm{y}=1,148+0,0378 \times \mathrm{AC}+0,1252 \times \mathrm{DC}$ \\
\hline & $y=-10,57+0,0339 \times A C+0,1539 \times D C+0,0706 \times B+1,91 \times$ IV3 \\
\hline \multirow{3}{*}{ Diâmetro de caule } & $\mathrm{y}=2,733+2,088 \mathrm{x}$ AP \\
\hline & $\mathrm{y}=6,24+0,1359 \times \mathrm{AC}$ \\
\hline & $y=18+9,4 \times A P-0,231 \times B$ \\
\hline \multirow{2}{*}{ Área de copa } & $y=-6,056+9,312 \times A P$ \\
\hline & $\mathrm{y}=1,637+2,378 \times \mathrm{DC}$ \\
\hline
\end{tabular}

$\overline{\mathrm{AC}}=$ área de copa; $\mathrm{DC}=$ diâmetro de copa; $\mathrm{AP}=$ altura de planta; $\mathrm{B}=$ banda do azul; IV3 = Nir / Verde.

\subsection{Separação dos alvos}

O sensoriamento remoto por meio de Veículos Aéreos Não Tripulados se mostra uma boa ferramenta para a separação de alvos espectrais encontrados na área de estudo (Figura 2). Das plantas alvos predominantes encontrados, duas espécies estavam bem vigorosas (Figuras 2A e 2B), em fase de senescência (Figura 2C), e outras ainda como planta de goiabeira, palhada, solo descoberto e sombra (Figuras 2D, 2E, 2F e 2G). 


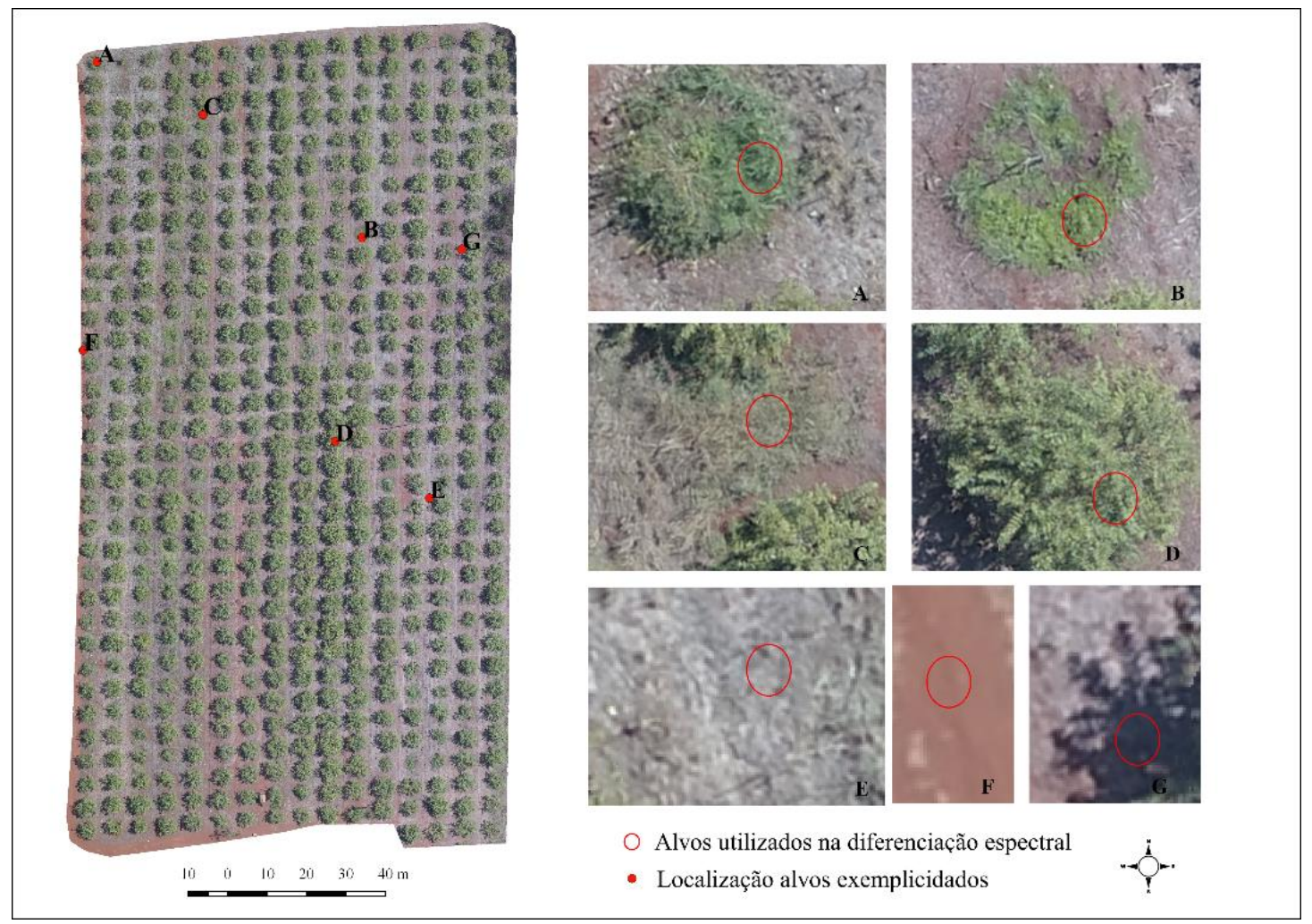

Figura 2: Amostras de alvos selecionados e localização na imagem no município de Santa Helena de Goiás, Goiás. A - planta daninha vigorosa, B - planta daninha vigorosa; $C$ - planta daninha senescendo; $D$ - planta de goiabeira; $E$ - palhada; $F$ - solo descoberto e $G$ - sombra de planta.

A separação espectral de alvos é uma pratica muito eficiente para um melhor manejo das atividades nos pomares, como exemplo o controle seletivo de plantas daninhas ou ainda em áreas selecionadas apenas com a infestação das mesmas. A prática de aplicação localizada de herbicidas visa economia de produtos, além de um manejo mais sustentável. Além disso, em uma segunda aplicação, a diferenciação da palhada para a planta daninha permite um manejo em somente aquelas cuja o herbicida não foi efetivo. Para os pomares de goiaba a separação permite a seleção de todas as plantas através de um algoritmo de classificação supervisionada, cuja permite em seguida analisar a área de copa de cada planta e sua sanidade (Figura 3).

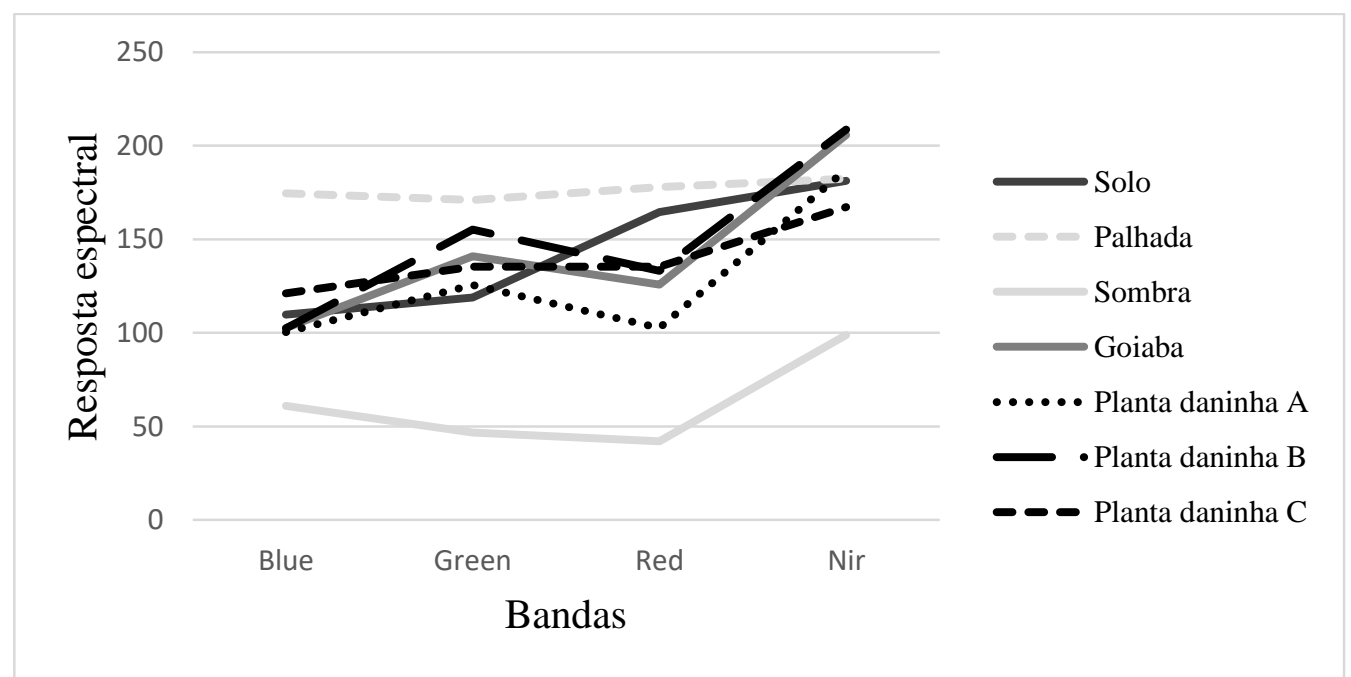

Figura 3: Curva de resposta espectral dos alvos selecionados em pomar de goiabeira no município de Santa Helena de Goiás, Goiás. 
Uma planta daninha quando vai senescendo, ela passa a absorver mais o verde e o Nir, sendo o vermelho refletido com maior intensidade. Esta observação pode ser utilizada para verificar remotamente a necessidade de uma segunda aplicação de herbicidas, já que uma planta morta tem o comportamento espectral muito diferente de uma outra ainda sadia ou em processo de senescência.

Nem todas as bandas permitem uma separação das plantas de goiaba dos demais alvos, por meio da resposta espectral. Na faixa do infravermelho próximo e do azul, tem-se um comportamento espectral semelhante à algumas plantas daninhas. A goiabeira não pode ser diferenciada da planta daninha B na banda do Nir e da planta daninha A e B na banda do azul. Entretanto a banda do vermelho e do verde permitem a separação inconfundível das plantas daninhas A e B. Todavia, as diferentes espécies de plantas daninhas, quase sempre podem ser diferenciadas em alguma faixa do espectro eletromagnético, ou ainda em função da fase fenológica da planta (Figura 3).

Observa-se que na faixa do vermelho é possível separar a maior quantidade de alvos. A sombra e a palhada apresentam respostas bem distintas em várias partes do espectro eletromagnético, sendo estes dois alvos mais facilmente detectáveis na área de estudo. O Nir é a única faixa cuja palhada pode ser confundida com a planta daninha e o solo exposto.

\section{CONCLUSÃO}

A resposta espectral do dossel das plantas de goiabeiras e a medida de área de copa, pode ser utilizada para estimar parâmetros como altura de planta e diâmetro de caule, considerando aplicações do sensoriamento remoto com drones.

A combinação de variáveis agronômicas e das respostas espectrais tem-se uma melhora no $\mathrm{R}^{2}$ ajustado em relação ao uso das regressões lineares simples para estimar parâmetros como altura de planta e diâmetros de caule em goiabeiras.

A alta resolução espacial das imagens do drone, permite a separação de alvos dentro de áreas de pomares, diferenciando por meio da resposta espectral, as plantas de goiaba, de plantas daninhas, de palhada, de solo exposto e de sombra, bem como de um alvo com relação ao outro em geral.

\section{REFERÊNCIAS BIBLIOGRÁFICAS}

1. Colomina I, Molina P. Unmanned aerial systems for photogrammetry and remote sensing: a review. ISPRS J. Photogr Remote Sens. 2014 Jun; 92:9-97, doi: doi:10.1016/j.isprsjprs.2014.02.013.

2. Singh KK, Frazier AE. A meta-analysis and review of unmanned aircraft system (UAS) imagery for terrestrial applications. Int J Remote Sens. 2018 Jan; 39:5078-5098, doi: 10.1080/01431161.2017.1420941.

3. Sonka S. Big data: fueling the next evolution of agricultural innovation. J Innov Manag. 2016 May;4: 114-136, doi: 10.24840/2183-0606_004.001_0008.

4. Everingham Y, Sexton J, Skocaj D, Inman-Bamber G. Accurate prediction of sugarcane yield using a random forest algorithm. Agron Sustain Dev. 2016 Apr;36(2):27-36, doi: 10.1007/s13593-016-0364-z.

5. Wolfert S, Ge L, Verdouw C, Bogaardt Marc-Jeroen. Big data in smart farming - a review. Agric Syst. 2017 May;153: 69-80, doi:10.1016/j.agsy.2017.01.023.

6. Zhang Q. Precision agriculture technology for crop farming. CRC Press, Washington, USA; 2015. 360 p.

7. Hedley $\mathrm{C}$. The role of precision agriculture for improved nutrient management on farms. J Sci Food Agric. 2015 Jan;95(1):12-19, doi: 10.1002/jsfa.6734.

8. Robson A, Rahman MM, Muir JS, Saint A, Simpson C, Searle C. Evaluating satellite remote sensing as a method for measuring yield variability in Avocado and Macadamia tree crops. Adv Animal Biosc. 2017 Sep;8(2):498-504, doi:10.1017/S2040470017000954.

9. Wulfsohn D, Zamora FA, Téllez CP, Lagos IZ, García-Fiñana M. Multilevel systematic sampling to estimate total fruit number for yield forecasts. Precision agric. 2012 Sep;13(2):256-275, doi:10.1007/s11119-011-9245-2.

10. Best S, Salazar F, Bastias RM, Leon L. Crop load estimation model to optimize yield-quality ratio in apple orchards, Malus Domestica Borkh, Var. Royal Gala. J Inform Technol Agric. 2008 Aug;3(1):1118.

11. Wright AH, Nichols D, Embree C. Evaluating the accountability of trunk size and canopy volume models for determining apple tree production potential across diverse management regimes. Acta Hortic. 2005 Nov;707:237-243, doi:10.17660/ActaHortic.2006.707.30. 
12. Sengupta S, Lee WS. Identification and determination of the number of immature green citrus fruit in a canopy under different ambient light conditions. Biosyst Eng. 2014 Jan;117:51-61, doi:10.1016/j.biosystemseng.2013.07.007.

13. Dorj Ulzii-Orshikh, Lee M, Yun San-seok. An yield estimation in citrus orchards via fruit detection and counting using image processing. Comp Electr Agric. 2017 Aug;140:103-112, doi: 10.1016/j.compag.2017.05.019.

14. Bargoti S, Underwood JP. Image segmentation for fruit detection and yield estimation in apple orchards. J Field Robot. 2017 Feb;34(6):1039-1060, doi:10.1002/rob.21699.

15. Underwood JP, Hung C, Whelan B, Sukkarieh S. Mapping almond orchard canopy volume, flowers, fruit and yield using LiDAR and vision sensors. Comp Electr Agric. 2016 Nov;130:83-96, doi:10.1016/j.compag.2016.09.014.

16. Battude M, Bitar AA, Morin D, Cros J, Huc M, Sicre CM, Dantec VL, Demarez V. Estimating maize biomass and yield over large areas using high spatial and temporal resolution Sentinel-2 like remote sensing data. Remote Sens Environ. 2016 Oct:184:668-681, doi:10.1016/j.rse.2016.07.030.

17. Rahman MM, Robson AJ. A novel approach for sugarcane yield prediction using Landsat time series imagery: A case study on Bundaberg region. Adv Remote Sens. 2016 Jun:5(2):93-102, doi:10.4236/ars.2016.52008.

18. Ye X, Sakai K, Asada SI, Sasao A. Inter-relationships between canopy features and fruit yield in citrus as detected by airborne multispectral imagery. Am Soc Agric Biol Eng. 2008;51(2):739-751, doi:10.13031/2013.24371.

19. Kumar A, Singh KN, Lal B, Singh RD. Mapping of apple orchards using remote sensing techniques in cold desert of Himachal Pradesh, India. J Indian Soc Remote Sens. 2008 May;36(4):387-392, doi: 10.1007/s12524-008-0038-7.

20. Robson A, Rahman MM, Muir J. Using worldview satellite imagery to map yield in Avocado (Persea americana): a case study in Bundaberg, Australia. Remote Sens. 2017 Nov; 9(12):1223-1243, doi:10.3390/rs9121223.

21. Robson A, Rahman MM, Saint A. Evaluating satellite remote sensing as a method for measuring yield variability in Avocado and Macadamia tree crops. Adv Animal Biosc. 2017 Jul;8(2):498-504, doi:10.1017/S2040470017000954.

22. Peña JM, Torres-Sánchez J, Castro AI, Kelly M, López-Granados F. Weed mapping in early-season maize fields using object-based analysis of unmanned aerial vehicle (UAV) images. Plos One. 2013 Oct;8(10):1-11, doi:10.1371/journal.pone.0077151.

23. López-Granados F, Torres-Sánchez J, Serrano-Pérez A, Castro AI. Mesas-Carrascosa FJ, Peña JM. Early season weed mapping in sunflower using UAV technology: variability of herbicide treatment maps against weed thresholds. Precis Agric. 2016 Apr;17(2):183-199, doi:10.1007/s11119-015-9415-8

24. Lan Y, Thomson SJ, Huang Y, Hoffmann WC, Zhang H. Current status and future directions of precision aerial application for site-specific crop management in the USA. Comput electron agric. 2010 Oct;74(1):34-38, doi:10.1016/j.compag.2010.07.001.

25. Koger CH, Shaw DR, Watson CE, Reddy KN. Detecting late-season weed infestations in soybean (Glycine max). Weed Technol. 2003 Dec;17(4):696-704, doi:10.1614/WT02-122.

26. Pérez-Ortiz M, Peña JM, Gutiérrez PA, Torres-Sánchez J, Hervás-Martínez C, López-Granados F. A semi-supervised system for weed mapping in sunflower crops using unmanned aerial vehicles and a crop row detection method. Appl Soft Comput. 2015 Dec;37:533-544, doi:10.1016/j.asoc.2015.08.027.

27. Castaldi F, Pelosi F, Pascucci S, Casa R. Assessing the potential of images from unmanned aerial vehicles (UAV) to support herbicide patch spraying in maize. Precis Agric. 2017 Feb;18(1):76-94, doi:10.1007/s11119-016-9468-3.

28. Tamouridou AA, Alexandridis TK, Pantazi XE, Lagopodi AL, Kashefi AL, Moshou D. Evaluation of UAV imagery for mapping Silybum marianum weed patches. Int J Remote Sens. 2017 Jun;38:8-10, doi:10.1080/01431161.2016.1252475.

29. Torres-Sánchez J, López-Granados F, Castro AI, Peña-Barragán JM. Configuration and specifications of an unmanned aerial vehicle (UAV) for early site-specific weed management. Plos One. 2013 Mar;8(3):115, doi: 10.1371/journal.pone.0058210.

30. Zhu Z, Woodcock CE. Object-based cloud and cloud shadow detection in Landsat imagery. Remote Sens Environm. 2012 Mar;118:83-94, doi:10.1016/j.rse.2011.10.028.

31. Aboutalebi M, Torres-Rua AF, Kustas WP, Nieto H, Coopmans C, McKee M. Assessment of different methods for shadow detection in high-resolution optical imagery and evaluation of shadow impact on calculation of NDVI, and evapotranspiration. Irrig Sci. 2018 May;36:1-23, doi:10.1007/s00271-0180613-9. 
32. Hsieh YT, Wu ST, Chen CT, Chen JC. Analyzing spectral characteristics of shadow area from ads-40 high radiometric resolution aerial images. Int Arch Photogram Remote Sens Spatial Inform Sci. 2016;41:223-227, doi:10.5194/isprsarchives-XLI-B7-223-2016.

33. Visão geral de regressão dos melhores subconjuntos (Minitab), 2019. Disponível em https://support.minitab.com/pt-br/minitab/18/.

34. Arantes BHT, Arantes LT, Ventura MVA, Costa EM, Bessa MM, Baliza LM, Moraes VH. Uso de drones na atualização de área construída de imóveis urbanos. Sci Plena.2018; Out;14(10):105301, doi: 10.14808/sci.plena.2018.105301.

35. Deroco MG, Galo MLBT, Vieira BS. Detecting and mapping root-knot nematode infection in coffee crop using remote sensing measurements. IEEE J Select Top Appl Earth Observ Remote Sens. 2017 Aug;10(12):5395-5403, doi:10.1109/JSTARS.2017.2737618.

36. Montgomery DC, Runger GC. Estatística aplicada e probabilidade para engenheiros, 5. ed., LTC, Rio de Janeiro: LTC. 2012. 464 p. 